\title{
Visualizing Material Processing via Photoexcitation-Controlled Organic-Phase Aggregation-Induced Emission
}

\author{
Jian Gu, ${ }^{1}$ Bingbing Yue $\mathbb{D}^{2},{ }^{2}$ Glib V. Baryshnikov, ${ }^{3}$ Zhongyu Li, ${ }^{1}$ Man Zhang, ${ }^{1}$ Shen Shen, \\ Hans Ågren, ${ }^{4}$ and Liangliang Zhu $\mathbb{D}^{1}$ \\ ${ }^{1}$ State Key Laboratory of Molecular Engineering of Polymers, Department of Macromolecular Science, Fudan University, \\ Shanghai 200438, China \\ ${ }^{2}$ College of Science, University of Shanghai for Science and Technology, Shanghai 200093, China \\ ${ }^{3}$ Division of Theoretical Chemistry and Biology School of Biotechnology, KTH Royal Institute of Technology, \\ SE-10691 Stockholm, Sweden \\ ${ }^{4}$ Department of Physics and Astronomy, Uppsala University, Box 516, SE-751 20 Uppsala, Sweden
}

Correspondence should be addressed to Bingbing Yue; yuebingbing@usst.edu.cn and Liangliang Zhu; zhuliangliang@fudan.edu.cn

Received 17 February 2021; Accepted 11 May 2021; Published 7 June 2021

Copyright ( 2021 Jian Gu et al. Exclusive Licensee Science and Technology Review Publishing House. Distributed under a Creative Commons Attribution License (CC BY 4.0).

\begin{abstract}
Aggregation-induced emission (AIE) has been much employed for visualizing material aggregation and self-assembly. However, water is generally required for the preparation of the AIE aggregates, the operation of which limits numerous material processing behaviors. Employing hexathiobenzene-based small molecules, monopolymers, and block copolymers as different material prototypes, we herein achieve AIE in pure organic phases by applying a nonequilibrium strategy, photoexcitationcontrolled aggregation. This strategy enabled a dynamic change of molecular conformation rather than chemical structure upon irradiation, leading to a continuous aggregation-dependent luminescent enhancement (up to $\sim 200$-fold increase of the luminescent quantum yield) in organic solvents. Accompanied by the materialization of the nonequilibrium strategy, photoconvertible self-assemblies with a steady-state characteristic can be achieved upon organic solvent processing. The visual monitoring with the luminescence change covered the whole solution-to-film transition, as well as the in situ photoprocessing of the solid-state materials.
\end{abstract}

\section{Introduction}

Although organic luminogens tend to be monomeric in organic solvents with an invariant emission, they usually show distinct emission behavior in aggregated states [1-3]. Accordingly, a change of the luminescence properties upon aggregation can be used for visualizing material aggregation and self-assembly with different forms. Aggregation-induced emission (AIE) [4-11], referring to some type of organic luminophores that can reveal bright luminescence in aggregated or solid states by the suppression of the nonradiative decay, plays an important role in many fields of application, like displays and lighting [4-11], in information technology [12-15], and molecular probing [16-18]. However, water is largely required for the preparation of AIE aggregates (e.g., adding water into THF for preparing most of AIE aggregates [4-11, 19-21] or conversely adding THF into water for aggregating some water-soluble AIEgens $[22,23])$. This operation brings considerable limitation for numerous material processes, especially for those polymer materials that will perform sensitive nanoscale features by different solution processing. Therefore, we plan to develop AIE in pure organic phases (no phase transition) to break through the restriction of solvent type in visualizing the material processing, the task of which remains challenging but desired.

Recently, AIE materials that rely on specific chemical reactions have emerged [24-26], which make it possible to address the above hypothesis. Nevertheless, these cases have still been developed basically in the aqueous phase for biological usage [24-28]. The alternative way of producing an AIE effect by light can be more advantageous, because light stimulus is typically precise and rapid, and can provide contactless spatial and temporal control [29-33]. However, the difficulty to produce AIE in pure organic solvents by 


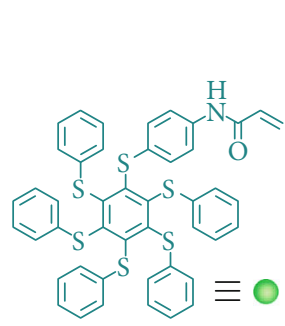

$\mathrm{H}$

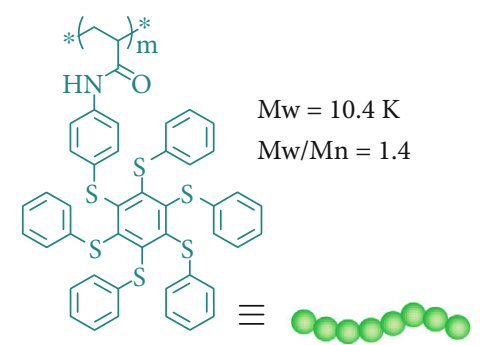

$\mathrm{PH}$

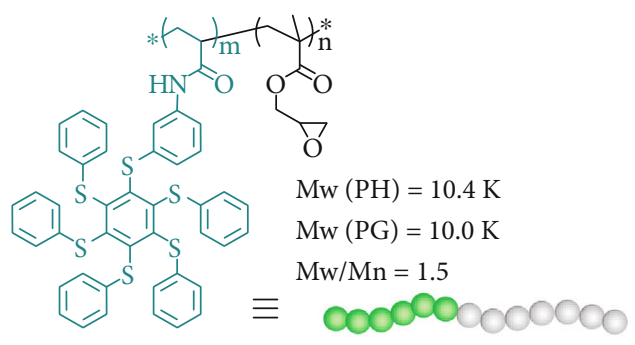

PH- $b$-PG

(a)
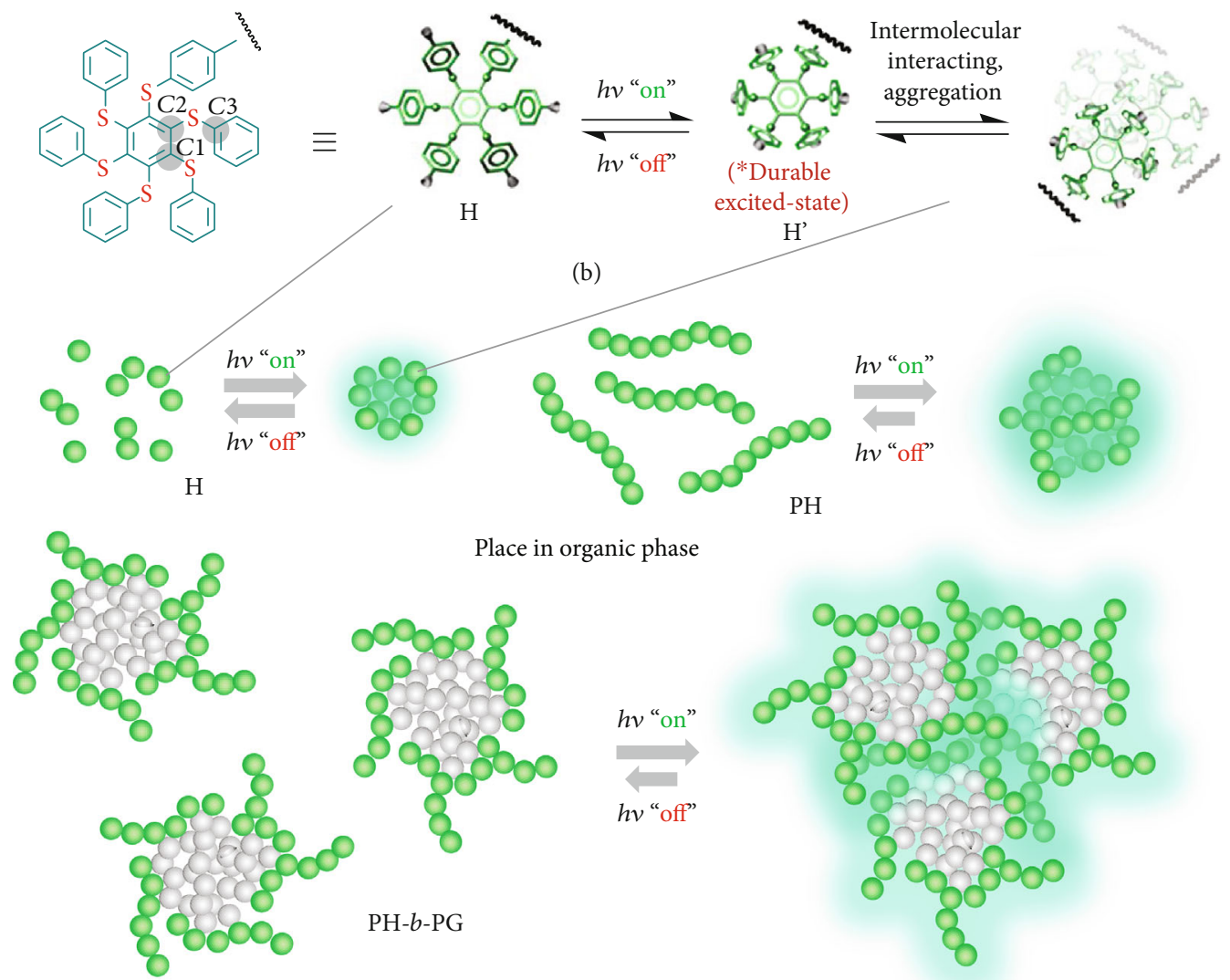

$\mathrm{PH}$

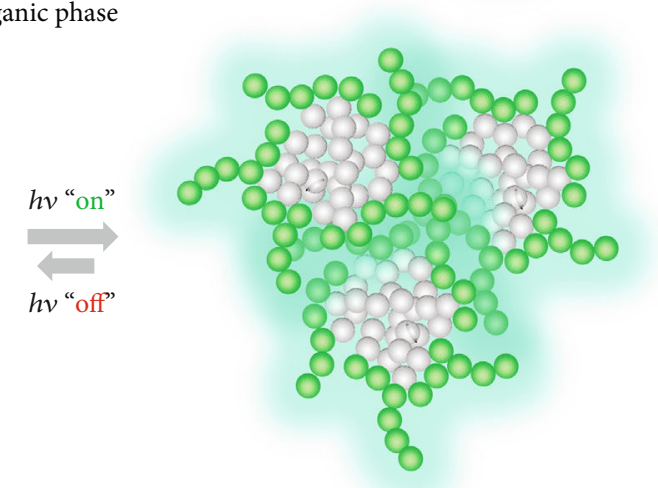

(c)

FIGURE 1: Outline for the photoexcitation-controlled organic-phase AIE: (a) chemical structures of monomer H, monopolymer PH, and block copolymer PH- $b$-PG based on hexathiobenzene; (b) the proposed conformational change upon photoexcitation of the hexathiobenzenebased skeleton. The related atoms were defined alongside the structure for the dihedral torsion analysis; (c) the illustration of photocontrolled organic-phase AIE accompanied by the molecular aggregation or morphological change of $\mathrm{H}, \mathrm{PH}$, and $\mathrm{PH}-b$ - $\mathrm{PG}$.

chemical or photochemical approaches could be the control of solubility difference between the reactant and the product. Instead, a photoexcitation-based physical process [34, 35], which can sufficiently utilize photons to facilitate the entire molecular motion, could be a suitable approach to change the molecular solubility before and after irradiation. Thus far, several nonequilibrium systems based on the photoexcitation principle have been developed by overcoming the ultrafast relaxation and dissipation of the excitation state energy [36-41]. Inspired by these nonequilibrium systems, we aim here to employ such a unique photophysical strategy into AIE-active luminescent materials to attend the aforementioned hypothesis.
In this work, hexathiobenzene-based small molecule, monopolymer, and block copolymer $(\mathrm{H}, \mathrm{PH}$, and $\mathrm{PH}-b$ - $\mathrm{PG}$, see chemical structure in Figure 1, as well as the synthetic details in SI) were used for the study of photoexcitationcontrolled organic-phase AIE. We take account of the fact that persulfurated aromatic molecules can serve as metalfree room-temperature phosphorescence emitters, for which an intersystem crossing process is environmentally adjustable. Due to this circumstance, persulfurated arenes can demonstrate unique aggregation-induced phosphorescence (AIP, an important class of AIE phenomena) [42, 43]. We fully utilized the asterisk-shaped molecular design of hexathiobenzene, which can show a significant conformational 
difference of molecular geometry between the ground and excited states. In addition, the durable excited-state of this luminophore guarantees a sufficient time lapse for molecular aggregation against excited-electron relaxation [44]. These factors can make it possible to achieve a photocontrollable wide-range organic-phase AIE. On the other hand, because the direct self-assembly (DSA) and the microphasesegregated feature of a block copolymer (BCP) is sensitive to the structure and solvent condition [45-47], we mainly focus on the BCP prototype before and after irradiation to highlight a visual monitoring of material self-assembly.

\section{Results}

2.1. AIE Behavior in Pure Organic Phases. As the hexathiobenzene-based skeleton contributed largely to the optical properties of these materials (evidenced by the similar band wavelength location in absorptional spectra among $\mathrm{H}$, $\mathrm{PH}$, and PH-b-PG, see Fig. S3), we firstly explore the photoluminescent property in different solvents. When PH- $b$-PG is placed in a variety of organic solvents, it exhibits a typical significant enhancement of the emission signal upon continuous UV irradiation (Figure 2(a)), accompanied by an increase of the luminescent quantum yield up to $\sim 200$-fold. This indicates a possible photocontrolled AIE behavior in the organic phases. Due to the solvent difference, the degree of the light-controlled aggregation as well as the solubility of the aggregates will differ, thus leading to different luminescence increments of the AIEgen. As it possesses the highest emission intensity in the 1,4-dioxane solvent after UV irradiation, we focused on this organic solvent for the further studies.

To access the possible AIE behavior with a photoexcitation principle, a study of the molecular structure of the compounds was initially performed. No changes in the results of the fundamental characterization were observed before and after irradiation (see the NMR and FT-IR spectra in Figs. S4-S9), indicating that the molecular structure of these species remains invariant and that the possibility of a photochemical reaction process is eliminated. However, the molecular aggregation behavior upon irradiation can be monitored by DLS and TEM studies. They show that the relatively well-dispersed nanometer-sized parts (less than $20 \mathrm{~nm}$ in diameter) of $\mathrm{H}$ and $\mathrm{PH}$ form larger nanometersized aggregates (more than $90 \mathrm{~nm}$ in diameter) after irradiation (Figures 2(b)-2(e)). On the other hand, PH- $b-\mathrm{PG}$ can initially form micelles [48-50] with an average diameter of $95 \mathrm{~nm}$ in 1,4-dioxane, and the micelles can be crosslinked upon UV irradiation (Figures 2(f) and 2(g)).

It is likely that such an aggregation tendency is responsible for the AIE, as the nearly quenched emission of the three compounds was found to appear immediately upon irradiation, and the emission signal $(\sim 470 \mathrm{~nm})$ became stronger over time (Figures 3(a)-3(c)). This behavior is synchronously accompanied by a small change of the absorption band at $>400 \mathrm{~nm}$. Such a photocontrolled AIE effect can be well visualized (see the corresponding insert photographs in Figures 3(a)-3(c)). Interestingly, the emission of these solutions is self-recoverable after the removal of the irradi- ation (see the emission signal decreased quickly at $470 \mathrm{~nm}$, Fig. S10). Such reversibility further indicates a nonequilibrium photoexcitation feature. Due to entanglement, the recovery of the polymers ( $\mathrm{PH}$ and $\mathrm{PH}-b-\mathrm{PG})$ is slower than that of the monomer $\mathrm{H}$.

2.2. Mechanism Study. The mechanism of photoexcitationcontrolled aggregation of the hexathiobenzene-based monomer and polymers can be explored from two perspectives, namely, from the population of the long-lifetime excited states and from the molecular conformational change upon photoexcitation. The formation of singlet oxygen, indicated by the reduction in the absorption of a quencher (1.3-diphenylisobenzofuran, DPBF) [51, 52], reflects the triplet-state behavior of the hexathiobenzene skeleton of $\mathrm{H}$ (Figure 3(d)). However, as compared to the power-law trend in DPBF absorption decay in the presence of the classic porphyrin photosensitizer (platinum(II) octaethylporphine, PTOEP, see Figure 3(e)), the decay of DPBF absorption exhibits an exponential trend in the presence of $\mathrm{H}$ (Figure 3(d)). This indicates a continuous variation of the triplet-state behavior of hexathiobenzene [53] upon the photoexcitation-induced molecular aggregation process (see also a full comparison in Fig. S11). In addition, we also access the ground- and excited-state conformations of $\mathrm{H}$ by theoretical calculations. As shown in Figure 3(f), the average dihedral torsion $\theta$ (defined by those atoms labeled in Figure 1) of $\mathrm{H}$ in the excited-state conformation is much smaller than that in the ground-state conformation $\left(118^{\circ}\right.$ in the $S_{0}$ state $v s .93^{\circ}$ in the $S_{1}$ state and $91^{\circ}$ in the $T_{1}$ state, see also detailed geometric parameters in Table S1). This suggests that the phenyl substituents are positioned more perpendicularly to the plane of the inner benzene core upon photoexcitation, facilitating the molecular aggregation process.

The concentration-dependent irradiation experiment of $\mathrm{PH}-b$-PG further verified the above mechanism. Because high concentration is more beneficial to intermolecular interaction and collision than low concentration, it is conducive to the photoexcitation-controlled aggregation and the luminescence enhancement (Figures 4(a)-4(c)). On the other hand, when the temperature was reduced, the emission enhancement of these compounds became less sensitive upon irradiation (Figures 4(d) $-4(\mathrm{f})$ ), indicating that the molecular motion induced by photoexcitation can be inhibited at low temperature $(<150 \mathrm{~K})$.

2.3. Visualizing Organic Solution Processing. As BCPs can exhibit unique DSA upon solution processing [45-47], we utilize the aforementioned photoexcitation-controlled AIE behavior to visualize the DSA of PH- $b$-PG (Figure 5(a)). Films prepared by casting of PH- $b$-PG solution before and after irradiation onto mica substrates were placed in a closed jar for solvent annealing (Figure 5(b)). Different from the lack of nanoscale feature of $\mathrm{H}$ and PH (Fig. S12), a typical lamellar microphase-segregated feature could be observed from the DSA of PH- $b$-PG without irradiation, largely depending on the close volume fraction of the two blocks (see AFM image in Figure 5(c)) [54]. When DSA took place 

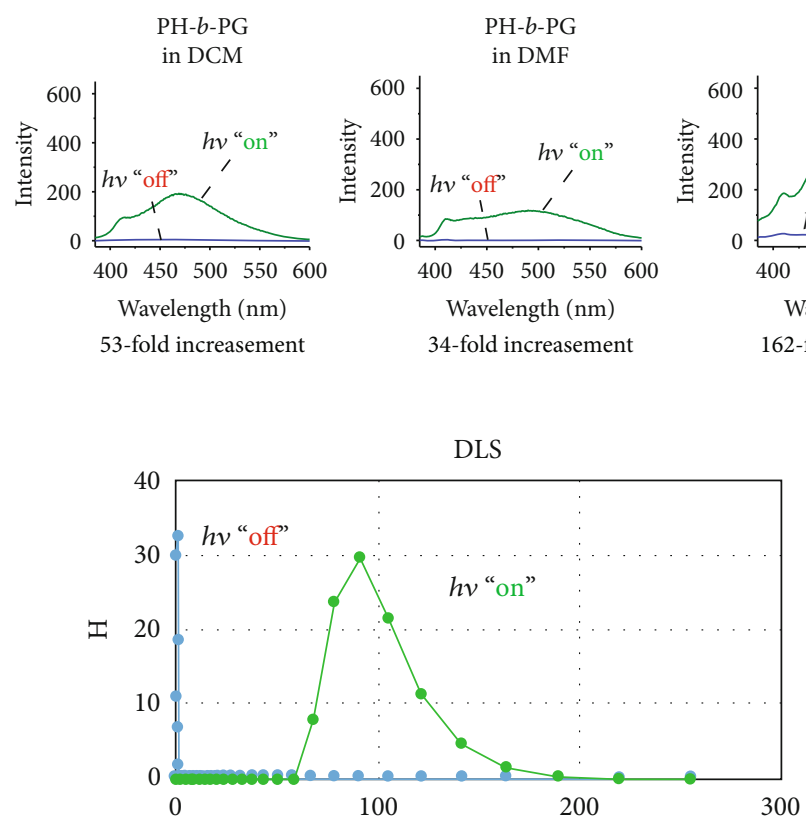

(b)

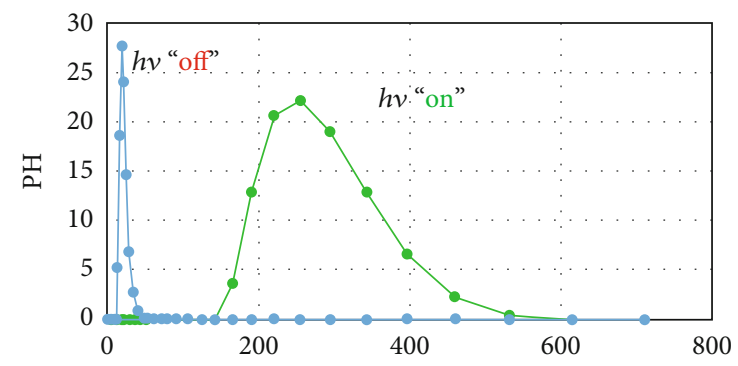

(d)

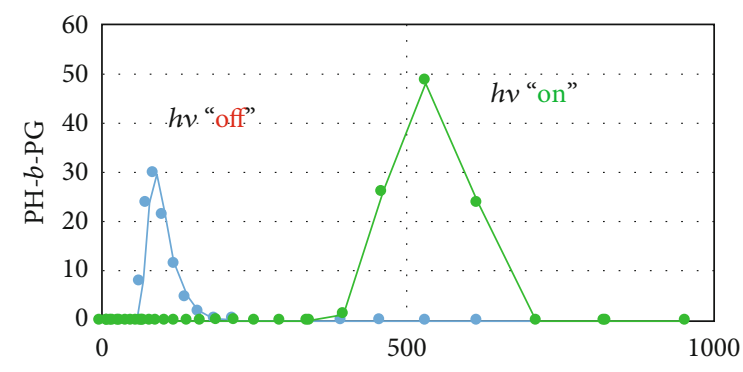

(f)
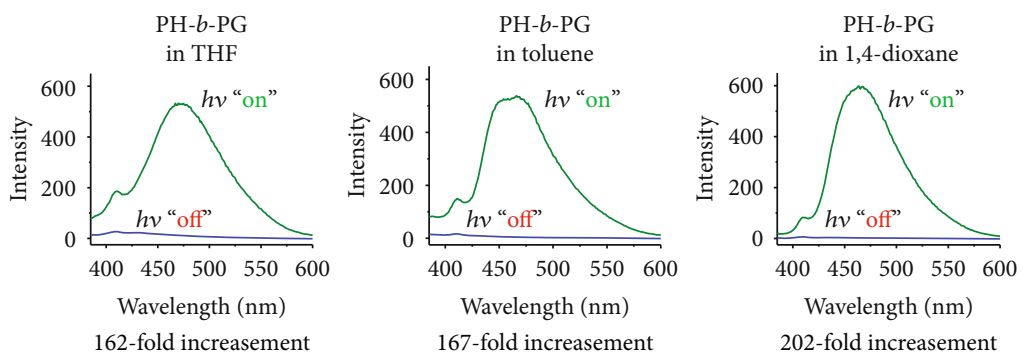

(a)

TEM

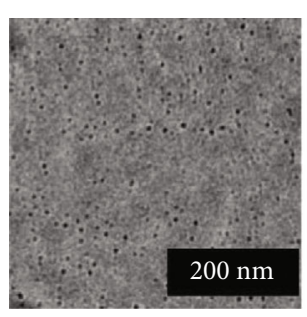

$h v$ "off"

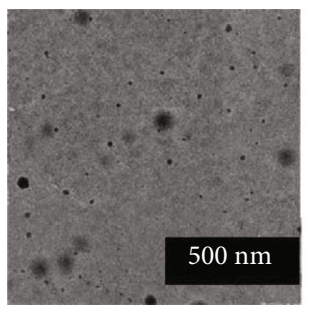

$h v$ "off"

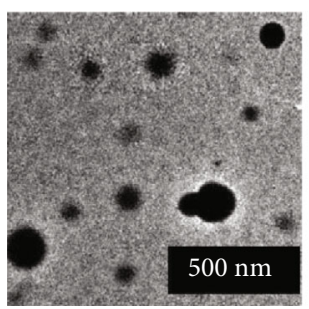

$h v$ "off"

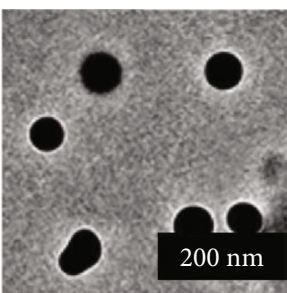

$h v$ "on"

(c)

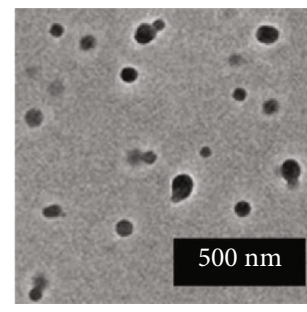

$h v$ "on"

(e)

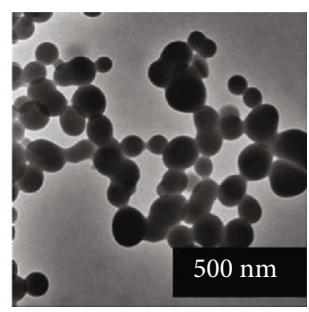

$h v$ "on"

(g)

Figure 2: AIE behavior in pure organic phases: (a) emission spectra of PH-b-PG placed in different organic solvents before and after irradiation for $90 \mathrm{~s}$. Spectra were collected upon $10 \mu \mathrm{M}$ of the hexathiobenzene unit at $298 \mathrm{~K}\left(\lambda_{\mathrm{ex}}=365 \mathrm{~nm}\right.$, measured in the same parameters). DLS results before and after irradiation for $90 \mathrm{~s}$ of (b) $\mathrm{H}$, (d) $\mathrm{PH}$, and (f) PH- $b$-PG, taken upon $10 \mu \mathrm{M}$ of the hexathiobenzene unit in 1,4-dioxane at $298 \mathrm{~K}$. TEM image of a sample of (c) H, (e) PH, and (g) PH- $b$-PG, prepared from a corresponding 1,4-dioxane solution before and after irradiation for $90 \mathrm{~s}$.

from the solution of PH-b-PG upon irradiation, the photocontrolled aggregation of the hexathiobenzene-based block will drive both of the two blocks as a whole for realignment, causing a photoconvertible microphase-segregated DSA from a lamellar to a cross-linked feature (Figure 5(c)). Both the DSAs before and after irradiation are amorphous at the molecular scale, evidenced by the broad XRD signals (Fig. S13).
2.4. Visualizing In Situ Photoexcitation Control. Upon on the organic solution processing with the visualization of the organic-phase AIE, we demonstrate more practical applications of these materials by photoexcitation-controlled molecular motion conducted directly in the film state under in situ irradiation. As the hexathiobenzene itself is a typical AIP luminophore, the film state of which, without additional doping matrix, initially exerts a strong emission in these 

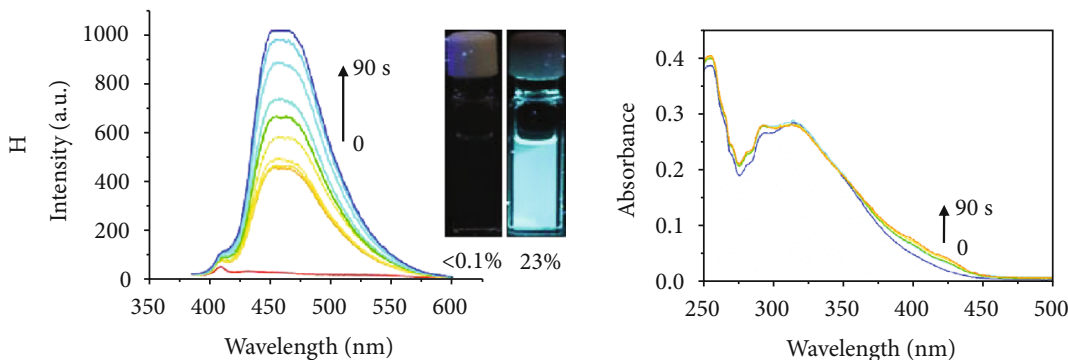

(a)
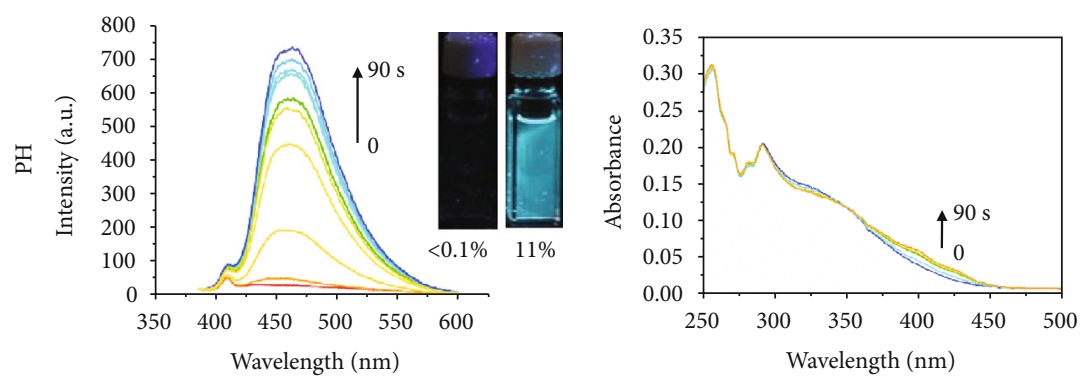

(b)

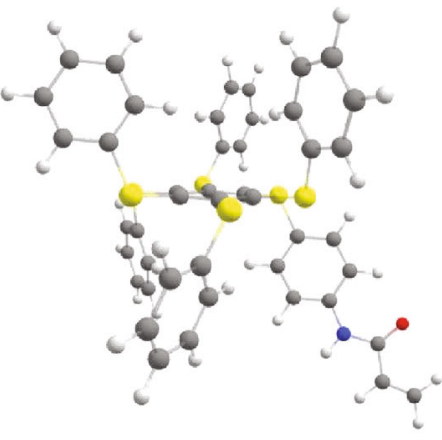

$S_{0}$ state, $\operatorname{Avg} \theta=118^{\circ}$

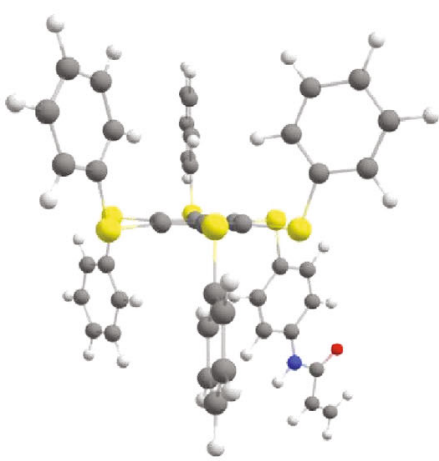

$S_{1}$ state, $\operatorname{Avg} \theta=93^{\circ}$

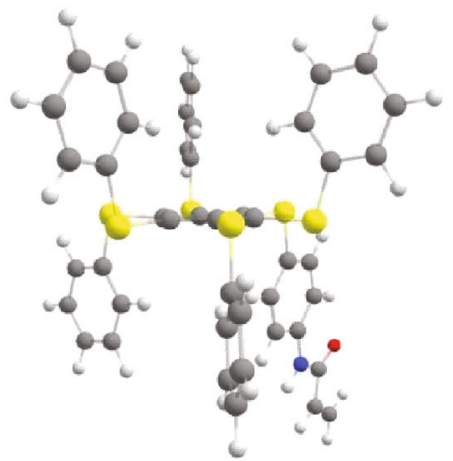

$T_{1}$ state, $\operatorname{Avg} \theta=91^{\circ}$

(f)

FIGURE 3: Mechanism study: continuous enhancement of emission and absorption spectra of (a) H, (b) PH, and (c) PH- $b$-PG in 1,4-dioxane with prolonged irradiation duration. The insert shows the corresponding photographs before and after irradiation under a UV lamp. The absorption and absorption intensity at $410 \mathrm{~nm}$ of DPBF in the presence of (d) H and (e) PTOEP versus the UV irradiation time for monitoring the singletoxygen formation and the triplet-state behavior. (f) Calculated geometry of the ground $\left(S_{0}\right.$ state) and excited states $\left(S_{1}\right.$ and $T_{1}$ states) of $\mathrm{H}$. 


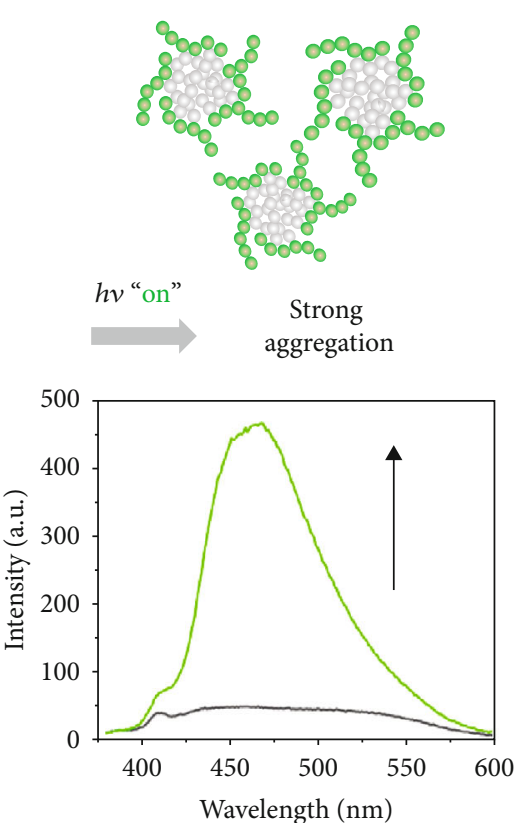

(a)

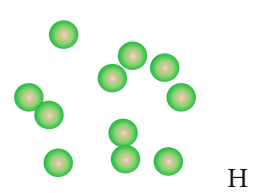

$h v$ "on"

$\longrightarrow$ No aggregation

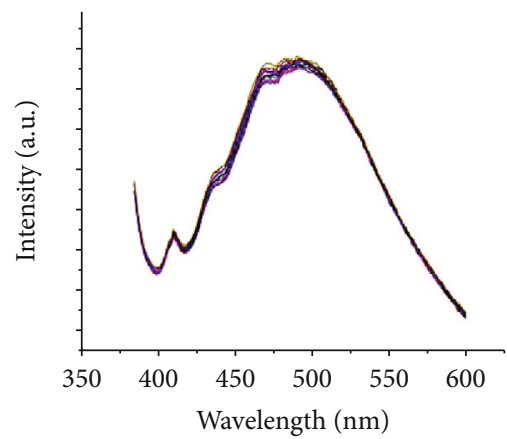

(d)
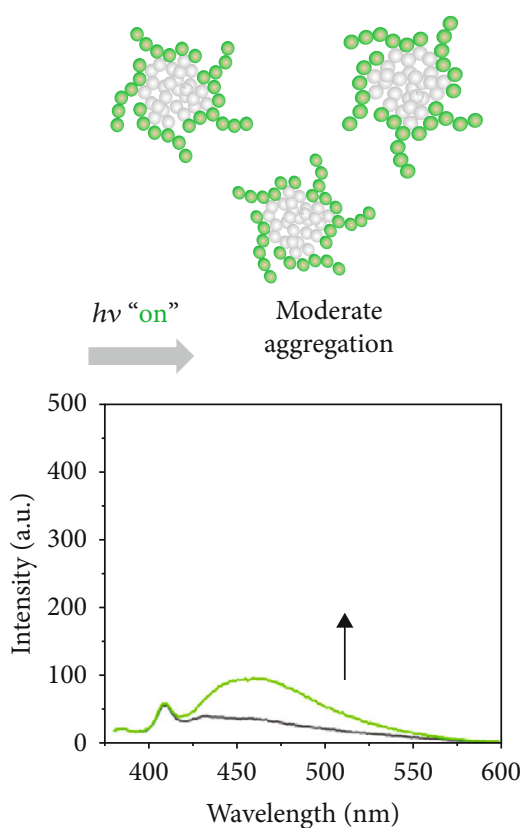

(b)

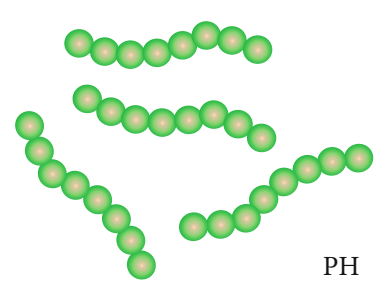

$\mathrm{PH}$
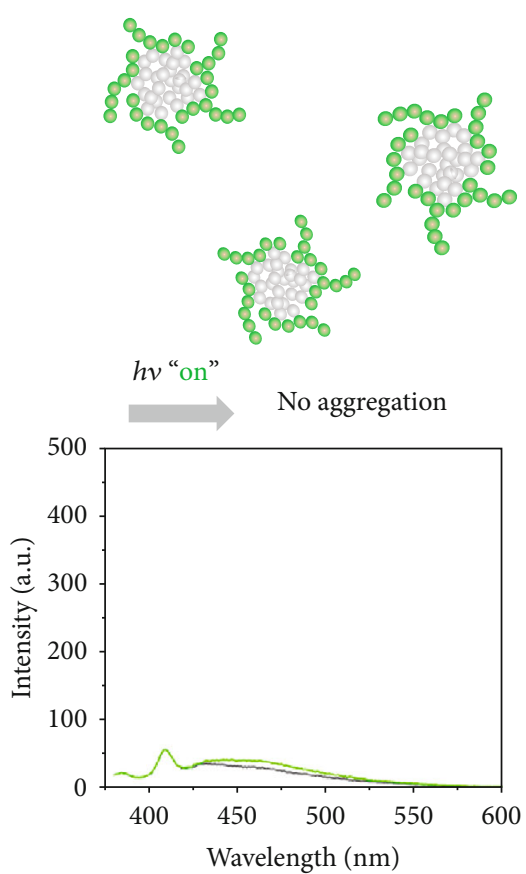

(c)

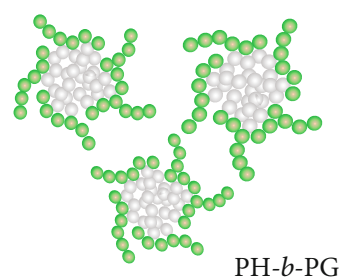

Placed in low temperature

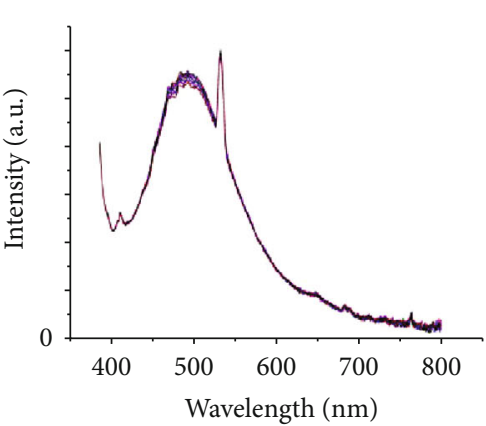

(e)

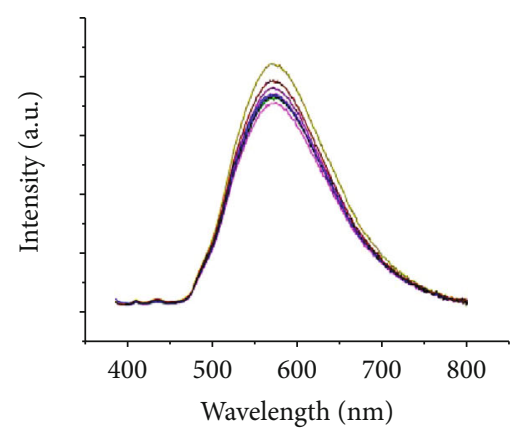

(f)

FIgURE 4: Molecular motion exploration: emission enhancements of PH- $b$-PG in 1,4-dioxane before and after irradiation for $10 \mathrm{~s}$ with the concentration of hexathiobenzene unit at (a) $0.1 \mathrm{mM}$, (b) $0.01 \mathrm{mM}$, and (c) $0.001 \mathrm{mM}$. Emission spectra of (d) $\mathrm{H}$, (e) $\mathrm{PH}$, and (f) $\mathrm{PH}-b$ PG in 1,4-dioxane with continuous UV irradiation at low temperature (less than $150 \mathrm{~K}$ ).

compounds (Figures 6(a)-6(c)). This is due to the fact that the molecules are already in a condensed state and thus inhibit the nonradiative decay. In these cases, a microsecond scale lifetime that signified an ambiguous phosphorescence emission can be well measured (Figures 6(d)-5(f)). After irradiation, the photoexcitation-controlled molecular motion caused the aggregation change, leading to molecules that turned into another condensed state with the emission reduced a little (Figures 6(a)-6(c)).

Different from the solution state, the self-recoverability of the film state of these materials and their emission signals are passivated (Fig. S14) because of the kinetic trapping 


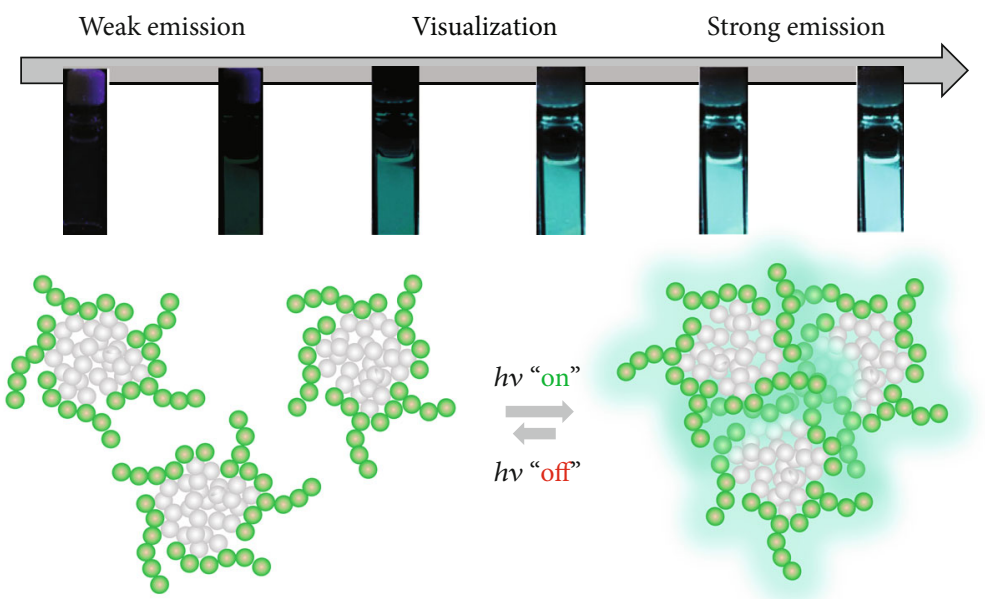

(a)
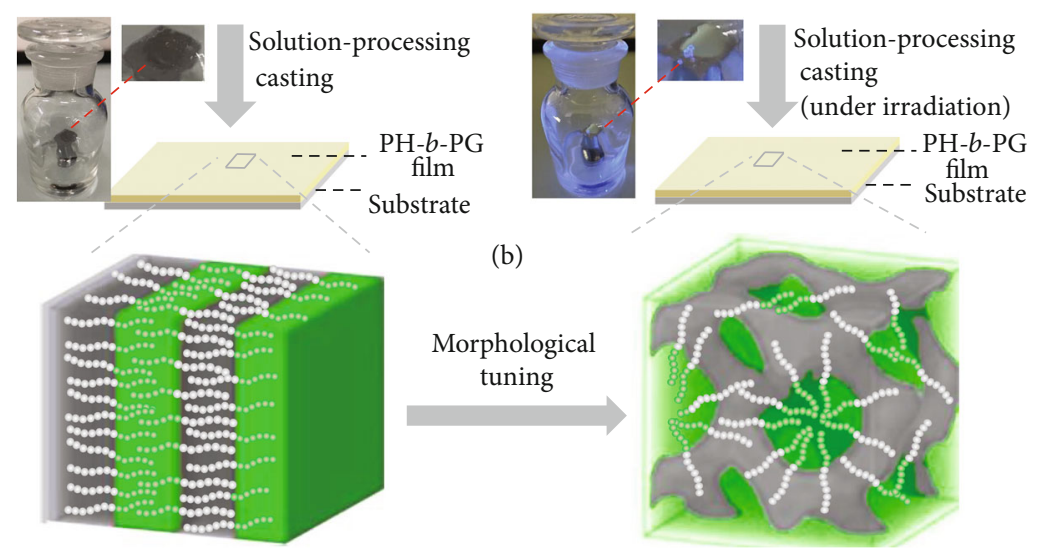

(b)
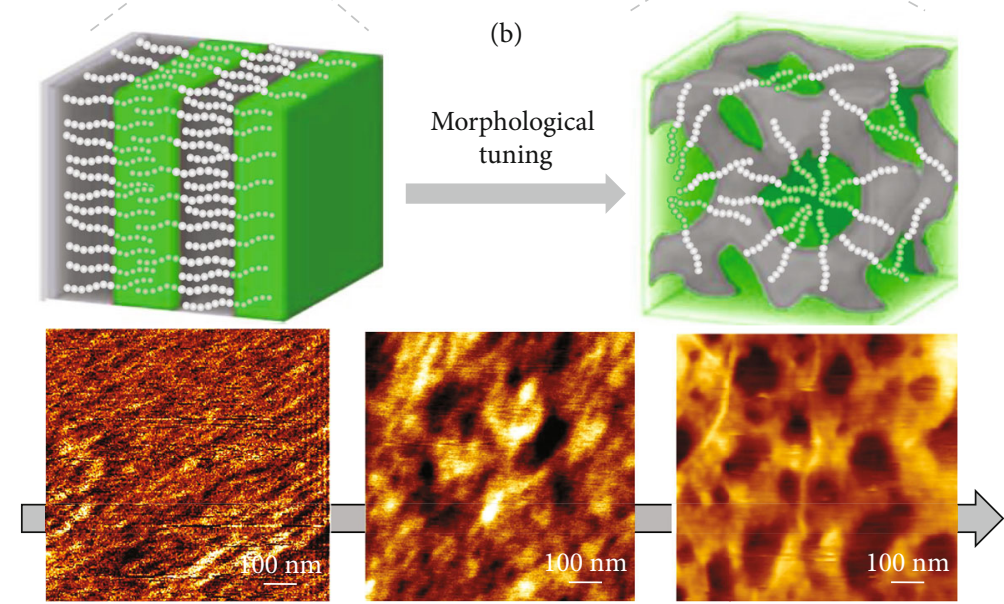

(c)

FIGURE 5: Visualizing organic solution processing: (a) illustration of the employment of photoexcitation-controlled AIE behavior to visualize the DSA of PH- $b$-PG with different morphologies, accompanied by a continuous luminescent enhancement upon irradiation; (b) the films prepared by casting of PH- $b$-PG solution before and after irradiation onto mica substrates and the photograph of the films placed in a closed jar with the 1,4-dioxane vapor for annealing; (c) corresponding AFM height images of the PH- $b$-PG films showing a photoconvertible microphase-segregated DSA from lamellar to a cross-linked feature upon irradiation.

effect in the solid state $[55,56]$. Compared with the monomer $\mathrm{H}$, some weight loss (ca. 5\%) of PH- $b$-PG before the thermal decomposition temperature is observed $\left(<200^{\circ} \mathrm{C}\right.$, Figure S15), which is usually related to the bond breaking of the polymer chain. However, we can find that such a weight loss of PH- $b$-PG was postponed after photoirradiation (Figure S15), showing an enhanced thermal stability upon the change of aggregation. This can also explain the irreversibility of the photoexcitation-controlled molecular motion in the solid state, a behavior of which is beneficial for a steady-state application.

Motivated by the observations reviewed above, we perform an application showcase of $\mathrm{PH}-b$-PG with photoirradiation. As hexathiobenzene is a luminophore with charge-transfer (CT) nature $[42,43]$, it is also sensitive to the polarity of the microenvironment [57]. The nonpolar block PG can affect the microenvironment of the hexathiobenzene luminophore upon the photoconvertible microphasesegregated self-assembly (referenced by the depiction in Figure 5(c)), leading to a further emission blue-shift in $\mathrm{PH}$ $b$-PG (Figure 6(c)) relative to the unshifted emission in $\mathrm{H}$ and $\mathrm{PH}$ (Figures 6(a) and 6(b)). In solution, we did not observe such an emission shift because the nonpolar block PG is relatively free. By employing this photocontrolling property of $\mathrm{PH}-b$-PG, a visualized photopatterning experiment, without additional doping matrix, can be performed. As shown in Figure 6(g), when the PH-b-PG film was alternately covered by masks, the regionally selective irradiation 


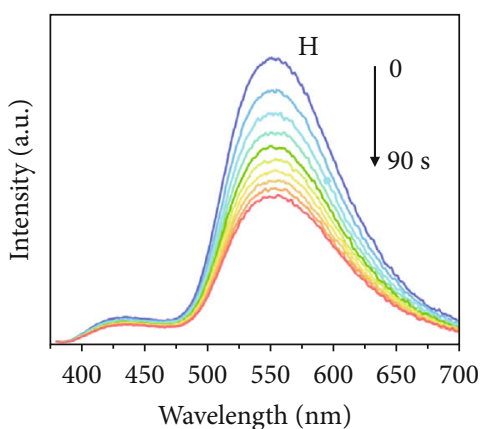

(a)

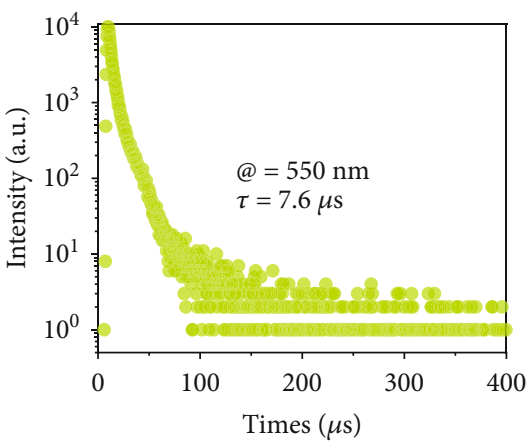

(d)

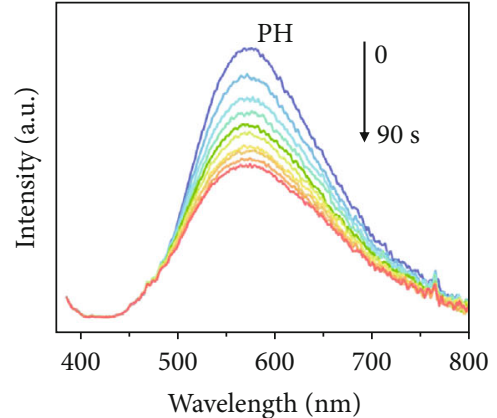

(b)

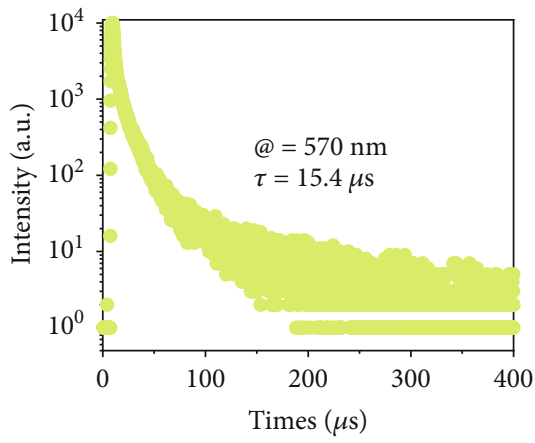

(e)

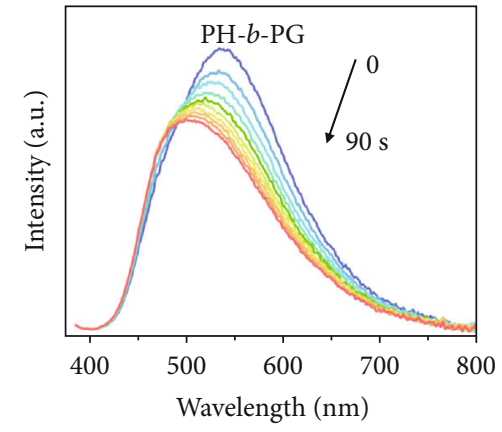

(c)

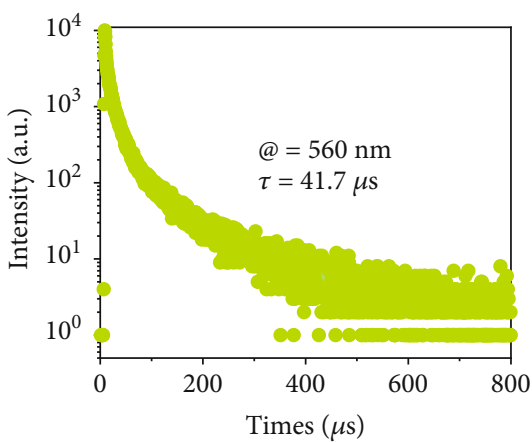

(f)
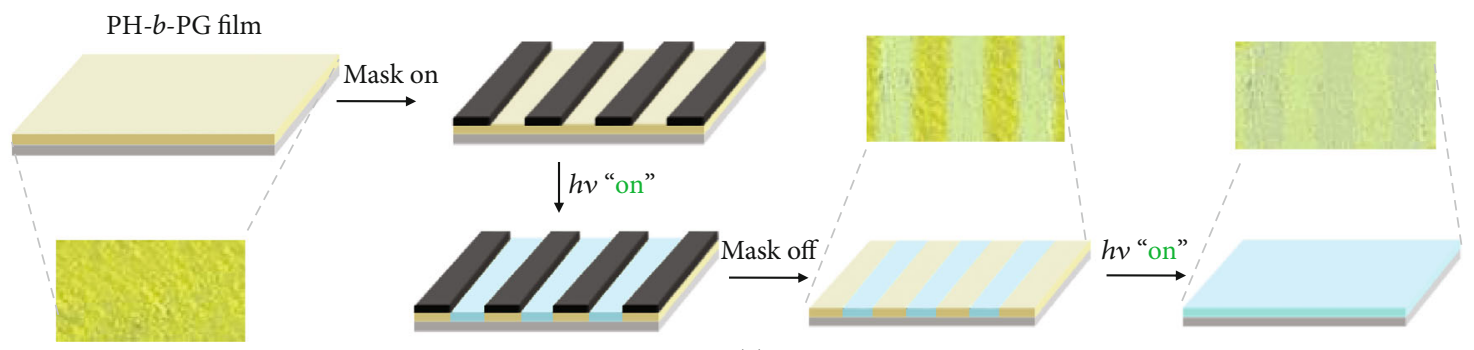

(g)

Figure 6: Visualizing in situ photoexcitation control: continuous change of emission of (a) $\mathrm{H}$, (b) PH, and (c) PH-b-PG in the film state with prolonged irradiation duration. Corresponding photoluminescent lifetime spectra of (d) H, (e) PH, and (f) PH- $b$-PG in the film state. (g) The visualized photopatterning experiment performed on a PH- $b$-PG film with regionally selective irradiation.

of the film gives a desired pattern showing emission stripes (see a cyan luminescence color relative to the yellow one alternate). The emission change induced by irradiation still continues after the masks are removed. This performance is advantageous for in situ photoexcitation-based control, indicating an application potential as functional materials with information processing $[58,59]$.

\section{Discussion}

This study presents a unique strategy for achieving organicphase AIE. It is based on a nonequilibrium process involving photoexcitation-induced molecular aggregation. The strategy is illustrated by an asterisk-shaped molecular design of the hexathiobenzene luminophore, which exerts a relatively large molecular conformational difference between its ground and excited states. On the other hand, the long lifetime of the excited-state guarantees a sufficient time lapse for molecular aggregation against the electron relaxation. As exemplified by the photoconvertible self-assembly accompanied by the organic-phase AIE, a visual monitoring of the solution processing of the materials with different morphologies could be achieved. Totally different from the previously reported examples of photoexcitation strategy with self-relaxation, a steady-state design conception was introduced here, moving forward the materialization of the photoexcitation-based physical strategy for material processing.

\section{Data Availability}

All data needed in the paper are present in the paper and in the Supplementary section. Additional data which are related to this paper may be requested from the authors.

\section{Conflicts of Interest}

The authors declare no conflict of interest. 


\section{Acknowledgments}

This work was supported by 2019 Natural Science Foundation of Shanghai (Grant No. 19ZR1471700), NSFC (21975046), and the National Key Research and Development Program of China (2017YFA0207700). G.B and H.Å. are thankful to Stiftelsen Olle Engkvist Byggmästare (contract no. 189-0223) for the support. The calculations were performed with computational resources provided by the High-Performance Computing Center North (HPC2N) in Umeå, Sweden, through the project "Multiphysics Modeling of Molecular Materials" SNIC 2020/3-29.

\section{Supplementary Materials}

Figure S1: synthetic route for $\mathrm{H}, \mathrm{PH}$, and $\mathrm{PH}-b$-PG. Figure S2: GPC spectra of PH and PH- $b$-PG. Figure S3: absorption spectra of $\mathrm{H}, \mathrm{PH}$, and $\mathrm{PH}-b$-PG. Spectra were collected upon $10 \mu \mathrm{M}$ of the hexathiobenzene unit in 1,4-dioxane at $298 \mathrm{~K}$. Figure S4: 1HNMR spectra of $\mathrm{H}$ in $\mathrm{CDCl} 3$ upon a continuous UV irradiation. Figure S5: FTIR spectra of $\mathrm{H}$ before and after UV irradiation for $3 \mathrm{~min}$. Figure S6: $1 \mathrm{HNMR}$ spectra of $\mathrm{PH}$ in $\mathrm{CDCl} 3$ upon a continuous UV irradiation. Figure S7: FTIR spectra of $\mathrm{PH}$ before and after UV irradiation for $3 \mathrm{~min}$. Figure S8: 1HNMR spectra of $\mathrm{PH}-b-\mathrm{PG}$ in $\mathrm{CDCl} 3$ upon a continuous UV irradiation. Figure S9: FTIR spectra of PH-b-PG before and after UV irradiation for $3 \mathrm{~min}$. Figure S10: the emission changes of $\mathrm{H}, \mathrm{PH}$, and $\mathrm{PH}-b$-PG in 1,4dioxane after irradiation for $90 \mathrm{~s}$, followed by the relaxation without irradiation for the same period. Table S1: selected torsion angles $(\theta$, deg.) for the different conformers of $\mathrm{H}$. Figure S11: UV-vis absorption spectra after irradiation at $365 \mathrm{~nm}$. Figure S12: AFM height images of (a) $\mathrm{H}$ and (b) PH. Figure S13: the XRD spectra of PH- $b$-PG before and after UV irradiation for $10 \mathrm{~min}$. Figure S14: the emission changes of $\mathrm{H}, \mathrm{PH}$, and $\mathrm{PH}-b$ - $\mathrm{PG}$ in the film state after irradiation for $90 \mathrm{~s}$, followed by the relaxation without irradiation for the same period. Figure S15: TGA of (a) $\mathrm{H}$ and (b) PH- $b$-PG before and after irradiation and (c) a partially magnified spectrum of (b). (Supplementary Materials)

\section{References}

[1] Y. Sagara and T. Kato, "Stimuli-responsive luminescent liquid crystals: change of photoluminescent colors triggered by a shear-induced phase transition," Angewandte Chemie, vol. 47, pp. 5175-5178, 2008.

[2] L. Zhu, X. Li, Q. Zhang et al., "Unimolecular photoconversion of multicolor luminescence on hierarchical self-assemblies," Journal of the American Chemical Society, vol. 135, no. 13, pp. 5175-5182, 2013.

[3] Y. Zhou, G. Baryshnikov, X. Li, M. Zhu, H. Ågren, and L. Zhu, "Anti-Kasha's rule emissive switching induced by intermolecular H-bonding," Chemistry of Materials, vol. 30, no. 21, pp. 8008-8016, 2018.

[4] J. Luo, Z. Xie, J. W. Lam et al., "Aggregation-induced emission of 1-methyl-1,2,3,4,5-pentaphenylsilole," Chemical Communications, vol. 18, pp. 1740-1741, 2001.

[5] B. K. An, D. S. Lee, J. S. Lee, Y. S. Park, H. S. Song, and S. Y. Park, "Strongly fluorescent organogel system comprising fibrillar self-assembly of a trifluoromethyl-based cyanostilbene derivative," Journal of the American Chemical Society, vol. 126, pp. 10232-10233, 2014.

[6] S. A. Fateminia, Z. Wang, C. C. Goh et al., "Nanocrystallization: a unique approach to yield bright organic nanocrystals for biological applications," Advanced Materials, vol. 29, no. 1, p. 1604100, 2017.

[7] H. Wei, Z. Zhao, C. Wei et al., "Antiphotobleaching: a type of structurally rigid chromophore ready for constructing highly luminescent and highly photostable europium complexes," Advanced Functional Materials, vol. 26, no. 13, pp. 20852096, 2016.

[8] B. Liu, H. Nie, X. Zhou et al., "Manipulation of charge and exciton distribution based on blue aggregation-induced emission fluorophors: a novel concept to achieve highperformance hybrid white organic light-emitting diodes," Advanced Functional Materials, vol. 26, no. 5, pp. 776-783, 2016.

[9] T. Weng, G. Baryshnikov, C. Deng et al., "A fluorescencephosphorescence-phosphorescence triple channel emission strategy for full-color luminescence," Small, vol. 16, no. 7, p. $1906475,2020$.

[10] X.-H. Wang, N. Song, W. Hou et al., "Efficient aggregation-induced emission manipulated by polymer host materials," Advanced Materials, vol. 31, no. 37, p. 1903962, 2019.

[11] Y. C. Liu, C. S. Li, Z. J. Ren, S. K. Yan, and M. R. Bryce, “Allorganic thermally activated delayed fluorescence materials for organic light-emitting diodes," Nature Reviews Materials, vol. 3, no. 4, p. 18020, 2018.

[12] Y. Su, S. Z. Phua, Y. Li et al., "Ultralong room temperature phosphorescence from amorphous organic materials toward confidential information encryption and decryption," Science Advances, vol. 4, p. 9732, 2018.

[13] Y. Gong, Y. Zhou, B. Yue et al., "Multiwavelength anti-kasha's rule emission on self-assembly of azulene-functionalized persulfurated arene," Journal of Physical Chemistry C, vol. 123, no. 36, pp. 22511-22518, 2019.

[14] Q. Wang, Q. Zhang, Q. W. Zhang et al., "Color-tunable singlefluorophore supramolecular system with assembly-encoded emission," Nature Communications, vol. 11, no. 1, p. 158, 2020.

[15] G. Chen, W. Li, T. Zhou et al., "Conjugation-induced rigidity in twisting molecules: filling the gap between aggregation-caused quenching and aggregation-induced emission," Advanced Materials, vol. 27, no. 30, pp. 4496-4501, 2015.

[16] Q. Miao, C. Xie, X. Zhen et al., "Molecular afterglow imaging with bright, biodegradable polymer nanoparticles," Nature Biotechnology, vol. 35, no. 11, pp. 1102-1110, 2017.

[17] H. Wu, Z. Chen, W. Chi et al., "Structural engineering of luminogens with high emission efficiency both in solution and in the solid state," Angewandte Chemie, International Edition, vol. 58, no. 33, pp. 11419-11423, 2019.

[18] X. Li, G. Baryshnikov, L. Ding et al., "Dual-phase thermally activated delayed fluorescence luminogens: a material for time-resolved imaging independent of probe pretreatment and probe concentration," Angewandte Chemie, International Edition, vol. 59, no. 19, pp. 7548-7554, 2020.

[19] Y. Hong, J. W. Y. Lam, and B. Z. Tang, "Aggregation-induced emission," Chemical Society Reviews, vol. 40, no. 11, pp. 53615388, 2011. 
[20] L. Zhu, X. Li, S. N. Sanders, and H. Ågren, "Unimolecular photopolymerization of high-emissive materials on cylindrical self-assemblies," Macromolecules, vol. 48, no. 15, pp. 50995105, 2015.

[21] H. Wu, C. Hang, X. Li et al., "Molecular stacking dependent phosphorescence-fluorescence dual emission in a single luminophore for self-recoverable mechanoconversion of multicolor luminescence," Chemical Communications, vol. 53, no. 18, pp. 2661-2664, 2017.

[22] D. Wang, H. Su, R. T. K. Kwok et al., "Rational design of a water-soluble NIR AIEgen, and its application in ultrafast wash-free cellular imaging and photodynamic cancer cell ablation," Chemical Science, vol. 9, no. 15, pp. 3685-3693, 2018.

[23] Z. X. Liu, Z. L. Wu, M. X. Gao, H. Liu, and C. Z. Huang, "Carbon dots with aggregation induced emission enhancement for visual permittivity detection," Chemical Communications, vol. 52, no. 10, pp. 2063-2066, 2016.

[24] G. Zhang, G. M. Palmer, M. W. Dewhirst, and C. L. Fraser, "A dual-emissive-materials design concept enables tumour hypoxia imaging," Nature Materials, vol. 8, no. 9, pp. 747-751, 2009.

[25] C. Y. Yu, H. Xu, S. Ji et al., "Radiosensitizers: mitochondrionanchoring photosensitizer with aggregation-induced emission characteristics synergistically boosts the radiosensitivity of cancer cells to ionizing radiation," Advanced Materials, vol. 29, p. 160617, 2017.

[26] D. Ding, K. Li, B. Liu, and B. Z. Tang, "Bioprobes based on AIE fluorogens," Accounts of Chemical Research, vol. 46, no. 11, pp. 2441-2453, 2013.

[27] Y. Yuan, C. Zhang, and B. Liu, "A photoactivatable AIE polymer for light-controlled gene delivery: concurrent endo/lysosomal escape and DNA unpacking," Angewandte Chemie, International Edition, vol. 54, no. 39, pp. 11419-11423, 2015.

[28] Y. Yuan, S. Xu, C. Zhang, and B. Liu, "Light-responsive AIE nanoparticles with cytosolic drug release to overcome drug resistance in cancer cells," Polymer Chemistry, vol. 7, pp. 3530-3539, 2016.

[29] H. Zhou, C. Xue, P. Weis et al., "Photoswitching of glass transition temperatures of azobenzene-containing polymers induces reversible solid-to-liquid transitions," Nature Chemistry, vol. 9, no. 2, pp. 145-151, 2017.

[30] L. Zhu, H. Tran, F. L. Beyer et al., "Engineering topochemical polymerizations using block copolymer templates," Journal of the American Chemical Society, vol. 136, no. 38, pp. 13381-13387, 2014.

[31] X. Chen, W. Zhao, G. Baryshnikov et al., "Engineering stable radicals using photochromic triggers," Nature Communications, vol. 11, no. 1, pp. 945-951, 2020.

[32] Y. Zhou, Q. Zou, J. Qiu, L. Wang, and L. Zhu, "Rational design of a green-light-mediated unimolecular platform for fast switchable acidic sensing," Journal of Physical Chemistry Letters, vol. 9, no. 3, pp. 550-556, 2018.

[33] S. Liu, X. Liu, J. Yuan, and J. Bao, "Multidimensional information encryption and storage: when the input is light," Research, vol. 2021, article 7897849, pp. 1-17, 2021.

[34] W. Chi, Q. Qiao, R. Lee et al., "A photoexcitation-induced twisted intramolecular charge shuttle," Angewandte Chemie, International Edition, vol. 58, no. 21, pp. 7073-7077, 2019.

[35] T. Muraoka, K. Kinbara, Y. Kobayashi, and T. Aida, "Lightdriven open-close motion of chiral molecular scissors," Jour- nal of the American Chemical Society, vol. 125, no. 19, pp. 5612-5613, 2003.

[36] V. Balzani, M. Clemente-León, A. Credi et al., "Autonomous artificial nanomotor powered by sunlight," Proceedings of the National Academy of Sciences of the United States of America, vol. 103, pp. 1178-1183, 2016.

[37] X. Jia, C. Shao, X. Bai et al., "Photoexcitation-controlled selfrecoverable molecular aggregation for flicker phosphorescence," Proceedings of the National Academy of Sciences of the United States of America, vol. 116, no. 11, pp. 4816-4821, 2019.

[38] J. Yang, X. Zhen, B. Wang et al., "The influence of the molecular packing on the room temperature phosphorescence of purely organic luminogens," Nature Communications, vol. 9, no. 1, pp. 840-849, 2018.

[39] L. Gu, H. Shi, M. Gu et al., "Dynamic ultralong organic phosphorescence by photoactivation," Angewandte Chemie, International Edition, vol. 57, no. 28, pp. 8425-8431, 2018.

[40] H. Zhang, L. du, L. Wang et al., "Visualization and manipulation of molecular motion in the solid state through photoinduced clusteroluminescence," Journal of Physical Chemistry Letters, vol. 10, no. 22, pp. 7077-7085, 2019.

[41] M. Gu, H. Shi, K. Ling et al., "Polymorphism-dependent dynamic ultralong organic phosphorescence," Research, vol. 2020, article 8183450, pp. 1-9, 2020.

[42] H. Wu, Y. Zhou, L. Yin et al., "Helical self-assembly-induced singlet-triplet emissive switching in a mechanically sensitive system," Journal of the American Chemical Society, vol. 139, no. 2, pp. 785-791, 2017.

[43] H. Wu, P. Zhao, X. Li et al., "Tuning for visible fluorescence and near-infrared phosphorescence on a unimolecular mechanically sensitive platform via adjustable $\mathrm{CH}-\pi$ interaction," ACS Applied Materials \& Interfaces, vol. 9, no. 4, pp. 3865-3872, 2017.

[44] K. Glusac, M. E. Köse, H. Jiang, and K. S. Schanze, "Triplet excited state in platinum-acetylide oligomers: triplet localization and effects of conformation," The Journal of Physical Chemistry B, vol. 111, no. 5, pp. 929-940, 2007.

[45] C. Tang, E. M. Lennon, G. H. Fredrickson, E. J. Kramer, and C. J. Hawker, "Evolution of block copolymer lithography to highly ordered square arrays," Science, vol. 322, no. 5900, pp. 429-432, 2008.

[46] B. Jung, P. Satish, D. N. Bunck, W. R. Dichtel, C. K. Ober, and M. O. Thompson, "Laser-induced sub-millisecond heating reveals distinct tertiary ester cleavage reaction pathways in a photolithographic resist polymer," ACS Nano, vol. 8, no. 6, pp. 5746-5756, 2014.

[47] B. Yue, X. Jin, P. Zhao, M. Zhu, and L. Zhu, "Directed selfassembly of templatable block copolymers by easily accessible magnetic control," Small, vol. 15, no. 7, p. 1804572, 2019.

[48] Y. Mai and A. Eisenberg, "A self-assembly of block copolymers," Chemical Society Reviews, vol. 41, pp. 5969-5985, 2012.

[49] J. B. Gilroy, A. D. Russell, A. J. Stonor et al., "An ironcyclopentadienyl bond cleavage mechanism for the thermal ring-opening polymerization of dicarba[2]ferrocenophanes," Chemical Science, vol. 3, no. 3, pp. 830-841, 2012.

[50] L. Yin, H. Wu, M. Zhu, Q. Zou, Q. Yan, and L. Zhu, "Sequential block copolymer self-assemblies controlled by metal-ligand stoichiometry," Langmuir, vol. 32, no. 25, pp. 6429-6436, 2016. 
[51] A. C. H. Ng, X. Li, and D. K. P. Ng, "Synthesis and photophysical properties of nonaggregated phthalocyanines bearing dendritic substituents," Macromolecules, vol. 32, no. 16, pp. 5292-5298, 1999.

[52] W. Spiller, H. Kliesch, D. Wöhrle, S. Hackbarth, B. Röder, and G. Schnurpfeil, "Singlet oxygen quantum yields of different photosensitizers in polar solvents and micellar solutions," Journal of Porphyrins and Phthalocyanines, vol. 2, no. 2, pp. 145-158, 1998.

[53] E. M. Gholizadeh, S. K. K. Prasad, Z. L. Teh et al., "Photochemical upconversion of near-infrared light from below the silicon bandgap," Nature Photonics, vol. 14, no. 9, pp. 585-590, 2020.

[54] F. S. Bates and G. H. Fredrickson, "Block copolymers-designer soft materials," Physics Today, vol. 52, no. 2, pp. 3238, 1999.

[55] E. Mattia and S. Otto, "Supramolecular systems chemistry," Nature Nanotechnology, vol. 10, no. 2, pp. 111-119, 2015.

[56] M. R. Molla, P. Rangadurai, L. Antony, S. Swaminathan, J. J. de Pablo, and S. Thayumanavan, "Dynamic actuation of glassy polymersomes through isomerization of a single azobenzene unit at the block copolymer interface," Nature Chemistry, vol. 10, no. 6, pp. 659-666, 2018.

[57] X. Li, G. Baryshnikov, C. Deng et al., "A three-dimensional ratiometric sensing strategy on unimolecular fluorescencethermally activated delayed fluorescence dual emission," Nature Communications, vol. 10, no. 1, p. 731, 2019.

[58] T. Leydecker, M. Herder, E. Pavlica et al., "Flexible nonvolatile optical memory thin-film transistor device with over 256 distinct levels based on an organic bicomponent blend," Nature Nanotechnology, vol. 11, no. 9, pp. 769-775, 2016.

[59] X. Yang, C. Vaswani, C. Sundahl et al., "Lightwave-driven gapless superconductivity and forbidden quantum beats by terahertz symmetry breaking," Nature Photonics, vol. 13, no. 10, pp. 707-713, 2019. 